Portland State University

PDXScholar

\title{
Transients in the Synchronization of Asymmetrically Coupled Oscillator Arrays
}

\author{
Carlos E. Cantos \\ Portland State University, ccantos@pdx.edu \\ David K. Hammond \\ Oregon Institute of Technology \\ J.J.P. Veerman \\ Portland State University, veerman@pdx.edu
}

Follow this and additional works at: https://pdxscholar.library.pdx.edu/mth_fac

Part of the Dynamic Systems Commons

Let us know how access to this document benefits you.

\section{Citation Details}

Cantos, C. E., Hammond, D. K., \& Veerman, J. J. P. (2016). Transients in the synchronization of asymmetrically coupled oscillator arrays. The European Physical Journal Special Topics, 225(6-7), 1199-1209.

This Post-Print is brought to you for free and open access. It has been accepted for inclusion in Mathematics and Statistics Faculty Publications and Presentations by an authorized administrator of PDXScholar. Please contact us if we can make this document more accessible: pdxscholar@pdx.edu. 


\title{
Transients in the Synchronization of Asymmetrically Coupled Oscillator Arrays
}

1 C. E. Cantos

Maseeh Department of Mathematics \& Statistics, Portland State University, Portland, OR e-mail: ccantos@pdx.edu

2 D. K. Hammond

Applied Mathematics Department, Oregon Institute of Technology, Wilsonville, OR

e-mail: david.hammond@oit.edu

3 J.J.P. Veerman

Maseeh Department of Mathematics \& Statistics, Portland State University, Portland, OR CQCN, Dept of Physics, University of Crete, Heraklion, Greece

e-mail: veerman@pdx.edu

\begin{abstract}
We consider the transient behavior of a large linear array of coupled linear damped harmonic oscillators following perturbation of a single element. Our work is motivated by modeling the behavior of flocks of autonomous vehicles. We first state a number of conjectures that allow us to derive an explicit characterization of the transients, within a certain parameter regime $\Omega$. As corollaries we show that minimizing the transients requires considering non-symmetric coupling, and that within $\Omega$ the computed linear growth in $N$ of the transients is independent of (reasonable) boundary conditions.
\end{abstract}

\section{Introduction}

In this paper we study the transient behavior of systems of asymmetrically coupled linear oscillators, following some initial perturbation. As the study of coupled linear oscillators is of great general importance in physics (see for example [27]), it may come as a surprise that even in the relatively simple case of a large (but finite) number of linear oscillators on the line with nearest neighbor coupling, we do not even know analytical expressions for the size of the transients! In this work we propose a solution to an old $([4,6])$ problem that concerns the stability of these systems as well as the quantitative description of the transients of those systems that are stable. Our theory is asymptotic in the sense that it becomes more accurate as the number of oscillators increases.

Even though the study of coupled oscillators and thus our results are of general interest, we develop our results by considering the specific problem of modeling automated control of vehicle platoons. We consider a long sequence of cars (or "agents") initially at rest. At $t=0$ the lead agent (or leader) acquires constant speed $v_{0}>0$. Each agent is equipped with sensors that perceive relative position and relative velocity of their neighbors, which is then used as feedback to determine its own acceleration. The initial nonzero velocity of the leader causes a perturbation which travels through- 


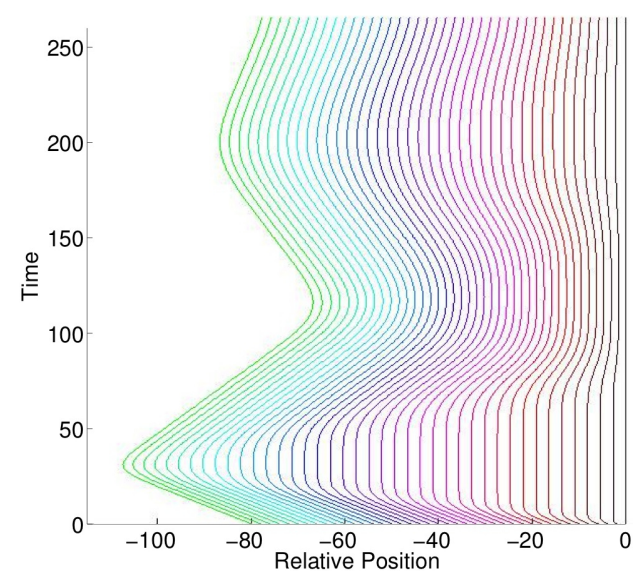

(a)

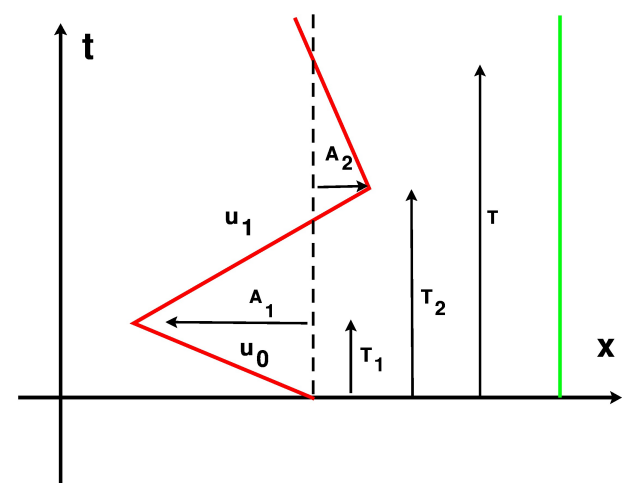

(b)

Fig. 1. (a) Numerical simulation with 40 agents. At $t=0$ the right most agent (the leader) starts moving to the right with constant velocity. The trajectories shown here are relative to the leader. (b) Schematic representation of the last agents orbit (red) and equilibruim position (dashed), relative to the leader. The vertical (green) segment on the far right represents the leader's orbit. We indicated the times $T_{i}$ at which the orbit has maximum amplitude whose absolute value equals $\left|A_{i}\right|$ and the time $T$ (the period) when it crosses its equilibrium position for the second time.

out the system (the transient), until the system, assuming it is asymptotically stable, approaches a state where all the agents have the same velocity $v_{0}$. A natural question is to find parameters for the feedback algorithm that minimize the transient, i.e. to ensure that the transient is bounded and decays quickly.

We describe the precise mathematical details of this system later, however as a qualitative introduction we show a simulation in Figure 1(a) where all agents are equally spaced and at rest at $t=0$, and the leader is given the fixed trajectory $x_{0}(t)=\max \left\{0, v_{0} t\right\}$ (i.e. constant velocity $v_{0}$ for $t>0$ ). This figure, as well as all following figures in this paper, shows the position of each agent relative to the leader, $x_{k}(t)-x_{0}(t)$. We focus our attention on the trajectory of the leftmost agent. Qualitatively, it can be seen that the perturbation (at $t=0$ ) of the leader's velocity creates a perturbation in the relative position that first propagates to the left, reflects off of the leftmost agent, propagates to the right, reflects off the leader and propagates to the left again. Also, the decaying, oscillating transient behavior of the leftmost agent can be clearly seen. The main result of this paper is to establish a precise characterization of the trajectory of the leftmost agent.

To understand quantitatively how a large system reacts when one agent (the leader) is kicked, we relate through a series of conjectures, the evolution of the system on the line to that of the system on the circle. This is known as imposing periodic boundary conditions, and it underlies much of Solid State Physics [27]. However in Solid State Physics, one typically deals with symmetric, undamped systems. Here we need an extension to the much more general case of non-symmetric, undamped systems.

Two remarks are in order here. The validity of the approach in the more restricted case does not guarantee success here. So we need to check our results carefully and this is done in Section 4. The second remark is that the more general theory may give rise to new phenomena. This indeed is the case. If the undamped, symmetric system is kicked, the perturbation typically travels with little distortion through the 
entire system. In the more general system, because of the damping, one might expect the perturbation to die out quickly. In fact, other things may happen. The system may be unstable, so that the perturbation grows indefinitely. Another possibility is more counter-intuitive. The system may be stable, but the transient perturbation grows exponentially large in the number of agents before they die out. This is called flock instability. Flock stable systems are where the perturbation grows less than exponential, and these are the ones with the smallest transients, and therefore these are the systems of primary interest for the applications we have in mind.

The paper is organized as follows. In Section 2 we describe the system of interacting agents and give some recent results. In Section 3 we derive the asymptotic behavior (for large $N$ ) of the transients. Finally, in Section 4, we present extensive numerical results that support the theory used in Section 3.

\section{Nearest Neighbor Flocks in $\mathbb{R}$}

We label the agents from 0 to $N$ (from right to left) and let $x_{k} \in \mathbb{R}$ be the position of the $k^{t h}$ agent. The feedback control is described by the function $f_{k}$ so that

$$
\ddot{x}_{k}=f_{k}\left(x_{k}-x_{k-1}, x_{k}-x_{k+1}, \dot{x}_{k}-\dot{x}_{k-1}, \dot{x}_{k}-\dot{x}_{k+1}\right) \text {, }
$$

This system is decentralized. This means (following [21] and [24]) that each agent's acceleration $\ddot{x}_{k}$ (apart from the leader's) depends only on positions and velocities relative to itself, i.e. on the differences $x_{k}-x_{i}$ and $\dot{x}_{k}-\dot{x}_{i}$ where $i$ is a neighbor of $k$. No absolute information is available to the agents. This definition has a long history in the literature (e.g. [4], [6], [5]).

In our model, the cars are all identical except for the leader, and the last vehicle which has no rear neighbor. In an initial (potentially nonlinear) model, we thus have all of the $f_{k}$ for $1 \leq k \leq N-1$ equal to a single function $f$. We fix the trajectory of the leader by $x_{0}(t)=\max \left\{0, v_{0} t\right\}$. We also impose the condition that there should be a fixed spacing $\Delta$ so that $f_{k}(-\Delta, \Delta, 0,0)=0$. This last condition implies that the above equations have so-called coherent motion solutions $x_{k}=v_{0} t+x_{0}-\Delta k$. We linearize this system around the coherent solution $x_{k}=x_{0}-\Delta k$ by substituting $x_{k}=x_{0}-\Delta k+\epsilon z_{k}$ into Equation 1. Working out to first order in $\epsilon$ and using $f_{k}(-\Delta, \Delta, 0,0)=0$, we obtain the linearized system :

$$
\ddot{z}_{k}=g_{x} \sum_{j=-1}^{1} \rho_{x, j} z_{k+j}+g_{v} \sum_{j=-1}^{1} \rho_{v, j} \dot{z}_{k+j}
$$

where $g_{x}, g_{v}, \rho_{x, j}$ and $\rho_{v, j}$ are constants (depending on the derivatives of $f$ ), and $\sum_{j=-1}^{1} \rho_{x, j}=\sum_{j=-1}^{1} \rho_{v, j}=0$.

The fact that $\rho_{x, j}$ and $\rho_{v, j}$ sum to zero follows from the system being decentralized. We see that Equation 2 may be written in matrix form as $\ddot{z}=g_{x} L_{x} z+g_{v} L_{v} \dot{z}$, where the row sums of the so-called graph Laplacian matrices $L_{x}$ and $L_{v}$ are zero.

We restrict ourselves to the case where $g_{x} \rho_{x, 0}$ and $g_{v} \rho_{v, 0}$ are both nonzero, and then assume without loss of generality that $\rho_{x, 0}=\rho_{v, 0}=1$ (otherwise we could rescale $g_{x}$ and $\left.g_{v}\right)$. Note then that $\rho_{x,-1}=-1-\rho_{x, 1}$ and $\rho_{v,-1}=-1-\rho_{v, 1}$, so all of the constants $\rho_{x, j}$ and $\rho_{v, j}$ are determined by specifying $\rho_{x, 1}$ and $\rho_{v, 1}$.

Completing the description of the linearized system requires specifying initial and boundary conditions. At $t=0$ the system is in equilibrium. For $t>0$ we specify the orbit of $z_{0}$ and the boundary condition at $z_{N}$ below. Here we have to make a choice for the boundary conditions. Two reasonable choices are given below. 
Definition 1 Let $S_{N}$ be the linearized system in Equation 2, where in addition

$$
z_{0}(t)=v_{0} \psi_{\epsilon}(t)
$$

where $\psi_{\epsilon}$ satisfies $\psi_{\epsilon}(t)=0$ for $t<-\epsilon$ and $\dot{\psi}_{\epsilon}(t)=1$ for $t>\epsilon$, and

$$
\ddot{z}_{N}=g_{x} \beta_{x}\left[-z_{N-1}+z_{N}\right]+g_{v} \beta_{v}\left[-\dot{z}_{N-1}+\dot{z}_{N}\right] .
$$

The parameters $\beta_{x}$ and $\beta_{v}$ are specified by one of the following choices:

1. Variable mass boundary conditions: $\beta_{x}=-\rho_{x,-1}$ and $\beta_{v}=-\rho_{v,-1}$

2. Regular boundary conditions: $\beta_{x}=\beta_{v}=1$

The first set of boundary conditions arises from simply leaving out the dependence on the relative velocity and position of the rear-neighbor. When $\rho_{x,-1}=\rho_{x, 1}$ and $\rho_{v,-1}=\rho_{v, 1}$, this leads to symmetric Laplacian matrices $L_{x}$ and $L_{v}$. For this reason that boundary condition is most often used (e.g. see [26], [10]). Notice though that physically this is akin to changing the mass of the last agent. In that sense the second set of boundary conditions (used by other authors, e.g. [4]) is more realistic.

The linear system $S_{N}$ has an eigenvalue zero with multiplicity 2 associated with the coherent motions. The system is asymptotically stable if all its other associated eigenvalues have negative real part (see [13]). However even if $S_{N}$ is asymptotically stable for all $N$, it is still possible to have the property that $\max _{t \in \mathbb{R}}\left|z_{N}(t)\right|$ grows exponentially with $N$. The notion of flock stability, given below, is defined to explicitly exclude this behavior (see also $[22,23,18]$ ).

Definition 2 (Flock stability) The system $S_{N}$ from Definition 1 is called flock stable if it is asymptotically stable and if $\max _{t \in \mathbb{R}}\left|z_{N}(t)\right|$ grows sub-exponentially in $N$.

Earlier characterizations of flocks concentrated on string stability, which is the variation of the relative distances between neighbors (e.g. [5], [12], [15], [11], [16], [17], $[9]$ ). This is hard to generalize for more complicated flocks in dimension 2 or higher. Also most of these papers, and many others ([9], [26]), in fact consider the size of frequency response as a measure of stability. While this is mathematically equivalent to the time-domain response, it is often difficult to explicitly calculate the time-response from the frequency response (see for instance [22], [23], [7], [8]).

We first state some previous results regarding the transients of the system $S_{N}$ in the time domain. Figure 1(b) shows a schematic description of the response when the leader leaves at $t=0$ with unit velocity. We have plotted the orbit of the last agent relative to that of the leader, or $z_{N}(t)-z_{0}(t)$. As can be seen in $1(\mathrm{a})$, the orbit of the last agent can be approximated by a piecewise constant "zig-zag" function that has its local extrema of $A_{k}$ at time $T_{k}$ for $k \geq 1$. A concise characterization of the transient is afforded by specifying the values of its local extrema $A_{k}$, its period $T$, and the attenuation coefficient $\alpha \equiv A_{3} / A_{1}$ (see Figure $1(\mathrm{~b})$ ). Some prior results valid for more limited parameter regimes are summarized as :

Theorem 1 i) ([25]) Consider the system $S_{N}$ with $\rho_{x, 1}=\rho_{v, 1}=-1 / 2$ and regular boundary conditions, and with $\psi_{\epsilon}(t)$ given by $\psi_{\epsilon}(t)=0$ for $t<0$ and $\psi_{\epsilon}(t)=t$ for $t \geq 0$. This system is flock stable and satisfies, in the limit as $N \rightarrow \infty$,

$$
\alpha \rightarrow 1 \quad \text { and } \quad \frac{A_{1}}{N} \rightarrow-\sqrt{\frac{2}{\left|g_{x}\right|}} \quad \text { and } \quad \frac{T}{N} \rightarrow \frac{4 \sqrt{2}}{\sqrt{\left|g_{x}\right|}}
$$

ii) ([22], [23], [18]) The system $S_{N}$ with $\rho_{x, 1}=\rho_{v, 1}=r$ and regular boundary conditions, is asymptotically stable and flock unstable for all $r \in(-1,0) \backslash\{-1 / 2\}$. 
Remark: We note that the case $\rho_{x, 1}=\rho_{v, 1}=r \in(-1 / 2,0)$ is particularly interesting since the real parts of the non-zero eigenvalues are bounded by $\max \left\{-\frac{g_{x}}{g_{v}}, g_{v}(1-\right.$ $2 \sqrt{|r|(1+r)})\}$, while when $r=-1 / 2$ the real parts tend to 0 as $N \rightarrow \infty$. Nonetheless the only flock stable case is the latter.

Our analysis relies on relating the evolution of $S_{N}$ to that of a similar system which has periodic boundary conditions denoted by $S_{N}^{*}$. Accordingly, we define

Definition 3 The system $S_{N}^{*}$ with periodic boundary satisfies, for all $k \in\{1, \cdots N\}$,

$$
\ddot{z}_{k}=g_{x} \sum_{j=-1}^{1} \rho_{x, j} z_{k+j}+g_{v} \sum_{j=-1}^{1} \rho_{v, j} \dot{z}_{k+j}
$$

where $z_{j}=z_{j+N}$ and $\sum_{j=-1}^{1} \rho_{x, j}=\sum_{j=-1}^{1} \rho_{v, j}=0$.

The main result from [2] is that if $S_{N}^{*}$ is stable then its solutions behave as traveling waves, moving in opposite directions, with velocities $c_{+}$and $c_{-}$(possibly unequal in magnitude). To establish this, an additional technical condition on the decay (as $N$ tends to infinity) of the expansion coefficients determined by the initial conditions is needed. This is expressed in terms of $a_{m}$ and $b_{m}$, where the total solution of the system $S_{N}^{*}$ has the form

$$
z_{k}(t)=\sum_{m=-N / 2}^{N / 2} a_{m} e^{i 2 \pi m k / N} e^{\nu_{m}-t}+\sum_{m=-N / 2}^{N / 2} b_{m} e^{i 2 \pi m k / N} e^{\nu_{m+} t}
$$

and $\nu_{m \pm}$ are the eigenvalues of the system matrix for $S_{N}^{*}$ (see [2] for details). Note that $a_{m}$ and $b_{m}$ may be calculated from the Fourier coefficients of $z_{k}(0)$ and $z_{k}^{\prime}(0)$ (considered as functions of $k$ ).

Theorem 2 (See [2]) Suppose the systems $S_{N}^{*}$ are asymptotically stable. Let $\alpha \in$ $(0,1)$. Suppose $q, M>0$ are such that both $\left|a_{m}\right|$ and $\left|b_{m}\right|$ are less than $N^{-1} M||^{-1-q}$. Then for large $N$, there are functions $f_{+}$and $f_{-}$and constants $K_{i}$ such that the solution $z_{j}(t)$ of $S_{N}^{*}$ satisfies

$$
\left|z_{k}(t)-f_{+}\left(k-c_{+} t\right)-f_{-}\left(k-c_{-} t\right)\right|<K_{1} N^{(2-q) \alpha-3} t+K_{2} N^{-\alpha q-1}
$$

The signal velocities $c_{ \pm}$(in terms of number of agents per unit time) are given by:

$$
c_{ \pm}=-\frac{g_{v}\left(1+2 \rho_{v, 1}\right)}{2} \pm \sqrt{\frac{g_{v}^{2}\left(1+2 \rho_{v, 1}\right)^{2}}{4}-\frac{g_{x}}{2}},
$$

where $c_{+}>0$ and $c_{-}<0$.

\section{Transients in Nearest Neighbor Systems}

The solutions shown in Figure 1 suggest that the transient following the initial perturbation of the leader can be understood to travel across the flock and reflect from the flock edge. Our analysis is based on tracking the path of this disturbance across the flock. Doing this requires understanding two phenomena - the velocity of the propagation of the disturbance, and the boundary conditions at the flock edge that will control the reflection. In addition, detailed analysis of the transient is only meaningful if the system is flock stable. 
We will first describe the path of a localized pulse, initially corresponding to the second derivative of the leader's orbit, across the system. For notational convenience we define $P_{N}$ to be the system, with coordinates $y_{k}(t)$, to satisfy the same equations as $S_{N}$ with the exception of the initial condition of the leader $y_{0}(t)$. For $P_{N}$, this is given by $y_{0}(t)=p(t) v_{0}$, where $p$ is a unit pulse satisfying $\int p(s) d s=1$ and having compact support. We will typically take $p(t)=\ddot{\psi}_{\epsilon}(t)$, where $\psi_{\epsilon}(t)$ is from equation (3). By linearity, this implies solutions of of $S_{N}$ can be obtained by integrating solutions of $P_{N}$ twice.

We base our quantitative analysis on the result given in Theorem 2 for the system with periodic boundary. As we have no analagous results for the system $S_{N}$, we pose a set of conjectures describing its stability, signal velocities, and boundary conditions.

Conjecture 1 If $S_{N}^{*}$ is asymptotically unstable then $S_{N}$ is asymptotically unstable or flock unstable.

Conjecture 2 If $P_{N}$ is stable and flock stable, then, away from the boundaries, the evolution of $P_{N}$ is as given in Theorem 2.

Conjecture 3 (Boundary Conditions) If $P_{N}$ is asymptotically stable and flock stable then its approximate traveling wave solution $\tilde{y}_{j}(t)$ may be chosen so that $\tilde{y}_{j}(t)$ satisfies the left boundary condition

$$
\left.\frac{\partial}{\partial j} \tilde{y}_{j}(t)\right|_{j=N}=0
$$

and the right boundary condition $\tilde{y}_{0}(t)=p(t)$.

It was proven in [2] that the system $S_{N}^{*}$ is asymptotically unstable unless $\rho_{x,-1}=$ $\rho_{x, 1}, g_{x} \rho_{x, 0}<0$, and $g_{v} \rho_{v, 0}<0$. By Conjecture $1, S_{N}$ cannot be flock stable unless these conditions hold. In particular if $\rho_{x, 1}=\rho_{v, 1} \in(-1,0) \backslash\left\{\frac{1}{2}\right\}$ it follows that $S_{N}$ is either unstable for large enough $N$ or flock unstable, consistent with Theorem 1 ii.

We now track the evolution of the unit pulse across $P_{N}$, obtaining an approximation $\tilde{y}_{N}(t)$ which consists of a sum of shifted and scaled (in amplitude) unit pulses. Note that the technical conditions on $a_{m}$ and $b_{m}$, as well as the details on the approximation error $\left|y_{N}(t)-\tilde{y}_{N}(t)\right|$, are precisely those from Theorem 2 .

Proposition 1 Suppose the systems $P_{N}$ are asymptotically stable and flock stable. Let $\alpha \in(0,1)$. Suppose there are $q, M>0$ such that $\left|a_{m}\right|$ and $\left|b_{m}\right|$ are less than $N^{-1} M|m|^{-1-q}$. Then for large $N$, there are constants $K_{i}$ and a function $\tilde{y}_{N}$ such that the trajectory $y_{N}(t)$ of the last agent of $P_{N}$ satisfies

$$
\left|y_{N}(t)-\tilde{y}_{N}(t)\right|<K_{1} N^{(2-q) \alpha-3} t+K_{2} N^{-\alpha q-1}
$$

where (with $C_{ \pm}$defined in Theorem 2)

$$
\tilde{y}_{N}(t)=\frac{c_{+}-c_{-}}{c_{+}} \sum_{k=0}^{\infty}\left(\frac{c_{-}}{c_{+}}\right)^{k} p\left(t-\frac{N}{c_{+}}-\left(\frac{1}{c_{+}}-\frac{1}{c_{-}}\right) k N\right) v_{0}
$$

Proof: By Conjecture 2, away from the boundaries, the solution $y_{j}(t)$ can be approximated within by $\tilde{y}_{j}(t)=f_{+}\left(t-j / c_{+}\right)+f_{-}\left(t-j / c_{-}\right)$. It remains to analyze the effect of the boundaries at $j=N$ and $j=0$ on the approximate solution. We give a reasoning analogous to the analysis in [3] (Appendix 2 to Chapter 5), but with two substantial modifications. First, the spatial variable is discrete and, second, the signal 
velocities depend on the direction. Because the system is linear we may use 1 instead of $v_{0}$ and multiply the solution we then obtain by $v_{0}$.

By the left boundary condition implied by Conjecture 3, we have

$$
-\frac{1}{c_{+}} f_{+}^{\prime}\left(t-\frac{N}{c_{+}}\right)-\frac{1}{c_{-}} f_{-}^{\prime}\left(t-\frac{N}{c_{-}}\right)=0 .
$$

Assume that $f_{-}$and $f_{+}$are continuous and integrate with respect to $t$ to get:

$$
c_{-} f_{+}\left(t-\frac{N}{c_{+}}\right)+c_{+} f_{-}\left(t-\frac{N}{c_{-}}\right)=0 .
$$

To simplify notation we will from now use:

$$
P \equiv\left(\frac{1}{c_{+}}-\frac{1}{c_{-}}\right) N \quad \text { and } \quad r \equiv \frac{c_{-}}{c_{+}}
$$

Substitute $s_{-}=t-N / c_{-}$into Equation 7 to get

$$
f_{-}\left(s_{-}\right)=-r f_{+}\left(s_{-}-P\right) .
$$

Conjecture 3 (at $j=0$ ) gives $f_{+}\left(s_{-}\right)+f_{-}\left(s_{-}\right)=p\left(s_{-}\right)$. Substitute Equation 9 into this and we get a recursion $f_{+}\left(s_{-}\right)=p\left(s_{-}\right)+r f_{+}\left(s_{-}-P\right)$ which implies:

$$
f_{+}\left(s_{-}\right)=p\left(s_{-}\right)+\sum_{k=1}^{\infty} r^{k} p\left(s_{-}-k P\right) .
$$

We note that for finite $s_{-}$, this is a finite sum because $c_{-}<0$ and therefore $\left(\frac{1}{c_{+}}-\frac{1}{c_{-}}\right)$ is positive, so that for $k$ sufficiently large, $p\left(s_{-}-k P\right)=0$.

On the other hand by using $s_{+}=s_{-}-P$, we see that Equation 9 gives:

$$
f_{+}\left(s_{+}\right)=-r f_{-}\left(s_{+}+P\right)
$$

We substitute this into $f_{+}\left(s_{+}\right)+f_{-}\left(s_{+}\right)=p\left(s_{+}\right)$and get $-r f_{-}\left(s_{+}+P\right)+f_{-}\left(s_{+}\right)=$ $p\left(s_{+}\right)$. Substituting $s_{-}$back this gives $f_{-}\left(s_{-}\right)=-r p\left(s_{-}-P\right)+r f_{-}\left(s_{-}-P\right)$, which implies:

$$
f_{-}\left(s_{-}\right)=-\sum_{k=1}^{\infty} r^{k} p\left(s_{-}-k P\right)
$$

Summing $f_{+}\left(t-j / c_{+}\right)$and $f_{-}\left(t-j / c_{-}\right)$gives the general approximate solution of the system (see Theorem 2)

$$
\tilde{y}_{j}(t)=p\left(t-\frac{j}{c_{+}}\right)+\sum_{k=1}^{\infty} r^{k}\left[p\left(t-\frac{j}{c_{+}}-k P\right)-p\left(\left(t-\frac{j}{c_{-}}\right)-k P\right)\right]
$$

Upon setting $j=N$, the terms telescope, and one obtains

$$
\tilde{y}_{N}(t)=\frac{c_{+}-c_{-}}{c_{+}} \sum_{k=0}^{\infty} r^{k} p\left(t-\frac{N}{c_{+}}-k P\right)
$$

Our main result may now be obtained by integrating these approximate solutions twice, and examining the resulting orbit which will be close to a "zig-zag" piecewise linear function such as illustrated in Figure 1 (b). We present our results (part $\underline{\mathrm{i}}$ below) in terms of the quantities $T$ and $A_{i}$ defined in the introduction. We also give a bound on the error of the difference between the approximate piecewise linear solution and the actual solution (part ii below). 
Theorem 3 Suppose the systems $S_{N}$ are asymptotically stable and flock stable. Let $\alpha \in(0,1)$. Suppose its solution $z_{k}(t)$ is such that its second derivative with respect to time satisfies Equation 4 where there are $q, M>0$ such that $\left|a_{m}\right|$ and $\left|b_{m}\right|$ are less than $N^{-1} M|m|^{-1-q}$.

For large $N$ and fixed $k_{0}$, the orbit $z_{N}(t)$ of the last agent of $S_{N}$ may be approximated by $\tilde{z}_{N}(t)$ with the following properties.

i: For $k \in\left\{1, \cdots k_{0}\right\}$

$$
\begin{aligned}
T_{k}= & \frac{N}{c_{+}}+(k-1)\left(\frac{1}{c_{+}}-\frac{1}{c_{-}}\right) N \\
A_{k}= & -\left(\frac{c_{-}}{c_{+}}\right)^{k-1} \frac{N v_{0}}{c_{+}} \\
T= & 2\left(\frac{1}{c_{+}}-\frac{1}{c_{-}}\right) N
\end{aligned}
$$

ii: For $0<t<T_{k_{0}}$ there are $K_{1}, K_{2}$ greater than 0 such that:

$$
\left|z_{N}(t)-\tilde{z}_{N}(t)\right|<K_{1} N^{(2-q) \alpha}+K_{2} N^{1-\alpha q}
$$

Remark: In the symmetric coupling case, the four $\rho$ 's are all equal to $-1 / 2$. So Equation 5 gives $c_{ \pm}= \pm \sqrt{\frac{\left|g_{x}\right|}{2}}$, and we recover Theorem 1 i.

Proof: The second derivative of $S_{N}$ is $P_{N}$ and Proposition 1 gives us its approximate solution $\tilde{y}_{n}(t)$. Therefore an approximate solution $\tilde{z}_{N}(t)$ of $S_{N}$ may be obtained by integrating $\tilde{y}_{n}(t)$. Its velocities $\dot{\tilde{z}}_{n}(t)$ are the integrals of $\tilde{y}_{N}(t)$. This tells us that at $t=T_{k} \equiv \frac{N}{c_{+}}+(k-1) P$ velocities $\dot{\tilde{z}}_{n}$ change.

The change of the velocity relative to the leader $\dot{\tilde{z}}_{N}(t)-v_{0}$ at $t=T_{k}$ is given by integrating the solution $\tilde{y}_{N}(t)$ of Proposition 1 around $t=T_{k}$. Using the notation of Equation 8:

$$
\int_{T_{k}-\epsilon}^{T_{k}+\epsilon} \tilde{z}_{N}(t) d t=(1-r) r^{k-1} v_{0}
$$

since $p(t)$ is a unit pulse. In the interval $\left[T_{k}+\epsilon, T_{k+1}-\epsilon\right]$ that velocity is then constant and it will be denoted by $u_{k}$. The initial velocity is $u_{0}=-v_{0}$ (with respect to the leader), and so we obtain the following recursion for the velocities $u_{k}$

$$
u_{k+1}=u_{k}+(1-r) r^{k-1} v_{0} \quad \text { and } \quad u_{0}=-v_{0}
$$

and this gives that $u_{k}=-r^{k} v_{0}$

Integrating once more (and noting that $\tilde{z}_{N}(0)=0$ ), we see that $\tilde{z}_{N}(t)-v_{0} t$ for $t>0$ is given by a continuous, piecewise affine function whose slope in the intervals $\left(T_{k}+\epsilon, T_{k+1}-\epsilon\right)$, with $T_{0}=0$, is given by $u_{k}$. We note that $T_{k+1}-T_{k}$ is proportional to $N$, so that $\frac{\epsilon}{T_{k+1}-T_{k}} \rightarrow 0$ as $N \rightarrow \infty$. Let us denote by $f_{k}$ the affine function, defined over the entire interval $\left(T_{k}, T_{k+1}\right)$, which agrees with $\tilde{z}_{N}(t)-v_{0} t$ on $\left(T_{k}+\epsilon, T_{k+1}-\epsilon\right)$. From the fact that $f_{k}\left(T_{k}\right)=A_{k}$ and $f_{k}^{\prime}(t)=u_{k}$ we deduce that

$$
t \in\left(T_{k}, T_{k+1}\right): f_{k}(t)=u_{k} t+\left(A_{k}-u_{k} T_{k}\right)
$$

From $f_{k}\left(T_{k+1}\right)=A_{k+1}$ we obtain

$$
A_{k+1}=A_{k}+u_{k}\left(T_{k+1}-T_{k}\right)=A_{k}+u_{k} P
$$

We furthermore know that in the interval $\left(0, T_{1}\right)$ the velocity $\dot{\tilde{z}}_{N}(t)-\dot{z}_{0}(t)$ equals $-v_{0}$ and that $\tilde{z}_{N}(0)-\dot{z}_{0}(t)=0$. This implies that $-v_{0} T_{1}=A_{1}$ and since we know $T_{1}$ we 

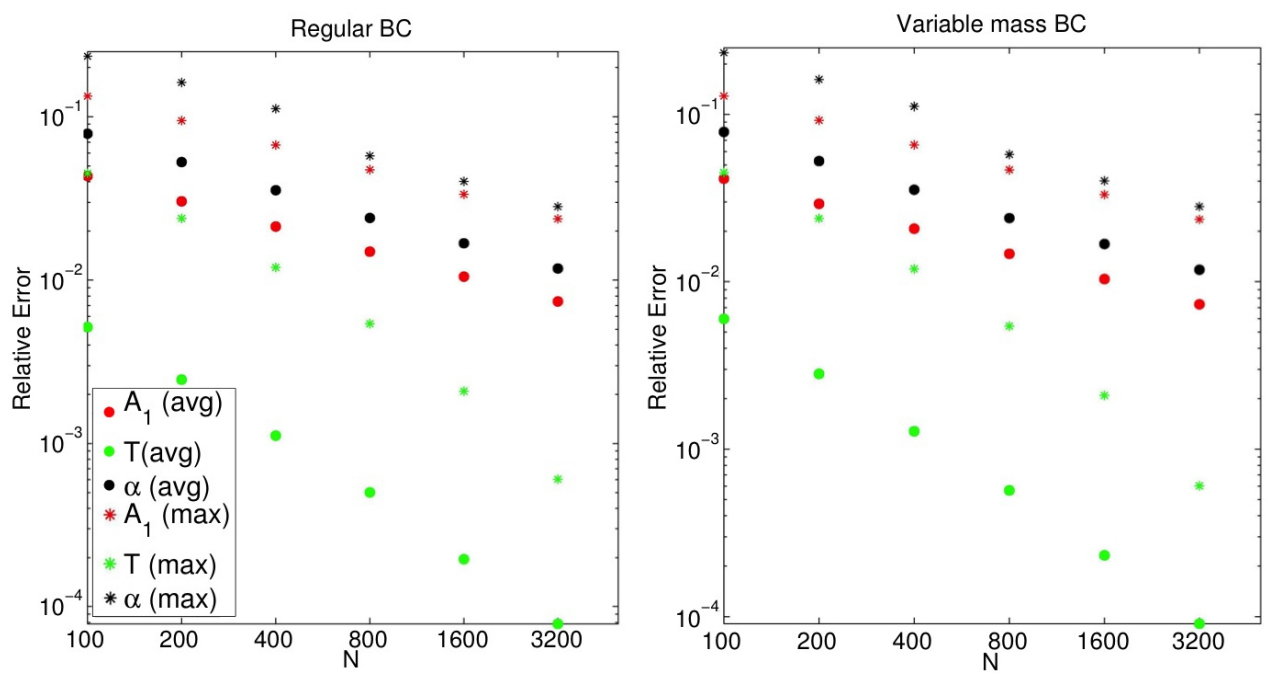

Fig. 2. Relative errors (predicted vs numerical results) for quantities $A_{1}, T$, and $\alpha$ describing the transient, as a function of $N$. Each plotted point corresponds to simulations using 30 different parameter values, as described in the text.

can solve for $A_{1}$ which gives $A_{1}=\frac{N v_{0}}{c_{+}}$. With that as initial condition, Equation 12 can easily be solved and this gives the second result.

The period $T$ can be calculated as the unique zero of the affine function $\tilde{z}_{N}(t)-v_{0} t$ given in Equation 11 in the interval $t \in\left(T_{2}, T_{3}\right)$. So we get

$$
T=T_{2}-\frac{A_{2}}{u_{2}} .
$$

We still have to establish the second part of the Theorem. By integrating Proposition 1 twice, we obtain that

$$
\left|z_{N}(t)-\tilde{z}_{N}(t)\right|<K_{5} N^{(2-q) \alpha-3} t^{3}+K_{6} N^{-\alpha q-1} t^{2}
$$

By item i, $T_{k_{0}}=O(N)$. Thus we obtain item ii by replacing $t$ by $N$.

Theorem 3 implies that the oscillations increase exponentially if $\left|c_{-}\right|>\left|c_{+}\right|$(from Equation 5). It is easy to check that this happens whenever $\rho_{v, 1}<-1 / 2$.

Corollary 1 To avoid exponential growth of the $\left|A_{i}\right|$, we need $\rho_{v, 1} \geq-\frac{1}{2}$.

\section{Numerical Results}

We tested our predictions by numerical simulations. For each each of the two boundary conditions in Definition 1, and each $N \in\{100,200,400,800,1600,3200\}$, we took a grid of 30 parameter values: $\rho_{v, 1} \in\{0,-0.1,-0.2,-0.3,-0.4,-0.5\}$ and $g_{v} \in\{-0.25,-0.50,-1,-2,-4\}$. This covers all the parameters still available for variation in Equation 2, since $\rho_{x, 1}=-1 / 2$ and $\left|g_{x}\right|$ can be set equal to 1 by rescaling time (i.e. by introducing $\tau=\sqrt{\left|g_{x}\right|} t$ ). For each parameter set we solved the system numerically using MATLAB's ode 45 solver then measured the amplitude $A_{1}$, the period $T$ (the average time elapsed between $T_{k}$ and $T_{k+2}$ ), and the attenuation $\alpha \equiv$ 

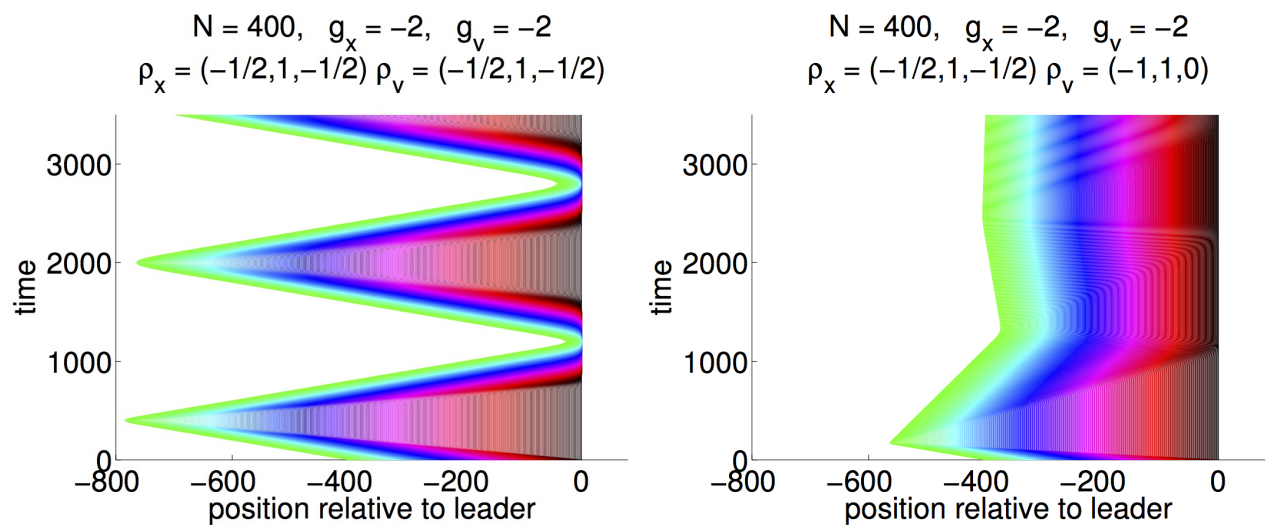

Fig. 3. Simulations for $N=400, g_{x}=g_{v}=-2$ and $\rho_{x, 1}=-1 / 2$, regular boundary conditions. In the first picture $\rho_{v, 1}=-1 / 2$, in the second $\rho_{v, 1}=0$. Each color represent the orbit of one of the 400 individual agents.

$A_{3} / A_{1}$ (see Figure $1(\mathrm{~b})$ ). We then compared these with the predicted values obtained in Theorem 3. The average, as well as the maximum (taken over the 30 parameter values described above), of the relative errors |measured-predicted|/|predicted| for each of these quantities are displayed in Figure 2 on a log-log plot.

Now that we know the transient as function of the parameters, one may seek to chose the parameters to minimize the transient. As a simple illustration of this direction, we consider a scalar measure $I_{E}$ of the transient that is proportional to the sum of the squares of the amplitudes $A_{k}$. By using Theorem 3 we get $I_{E} \equiv$ $\frac{1}{N^{2} v_{0}^{2}} \sum_{k=1}^{\infty} A_{k}^{2}=\frac{1}{c_{+}^{2}-c_{-}^{2}}$. As an example choose $g_{x}=g_{v}=-2$ and let $\rho_{x, 1}=-1 / 2$, and let $\rho_{v, 1}$ take the values $-1 / 2$ and 0 . We take $N=400$ and the leader leaves with velocity $v_{0}=1$. The simulations are exhibited in Figure 3 . The table below gives predictions for the various quantities. It is clear that the asymmetric choice $\rho_{v, 1}=0$ leads to much faster decay of the transient than the symmetric choice $\rho_{v,-1}=\rho_{v, 1}=-1 / 2$.

\begin{tabular}{|c|c|c|c|c|c|c|}
\hline & $c_{+}$ & $c_{-}$ & $A_{1}$ & $T$ & $\alpha$ & $I_{E}$ \\
\hline$\rho_{v, 1}=-1 / 2$ & 1 & -1 & 400 & 1600 & 1 & $\infty$ \\
\hline$\rho_{v, 1}=0$ & $1+\sqrt{2}$ & $1-\sqrt{2}$ & 166 & 2262 & 0.029 & 0.177 \\
\hline
\end{tabular}

\section{References}

1. P. Barooah, P. G. Mehta, J. P. Hespanha, Mistuning Based Control Design to Improve Closed Loop Stability Margin of Vehicular Platoons, IEEE Trans Autom Contr, Vol 54, 2100-2113, 2009.

2. C. E. Cantos, J. J. P. Veerman, D. K. Hammond, Signal Velocities in Oscillator Arrays, Submitted.

3. R. Courant, D. Hilbert, Methods of Mathematical Physics, Vol 2, John Wiley and Sons, 1962.

4. R. E. Chandler, R. Herman, E. W. Montroll, Traffic Dynamics: Studies in Car Following, Op. Res. 6, 165-184, 1958

5. K-C. Chu, Decentralized Control of High-Speed Vehicle Strings, Transp. Sci. 8, 361-384, 1974

6. R. Herman, E. W. Montroll, R. B. Potts, R. W. Rothery, Traffic Dynamics: Analysis and Stability in Car Following, Op. Res. 7, 86-106, 1959.

7. I. Herman, D. Martinec, Z. Hurák, M. Šebek, PDdE-based Analysis of Vehicular Platoons with Spatio-Temporal Decoupling, Proc 4th Workshop on Distributed Estimation and Control in Networked systems, Koblenz, Germany, 144-151, 2013.

8. D. Martinec, I. Herman, Z. Hurák, M. Šebek, Wave-absorber Vehicular Platoon Controller, ArXiv 1311.2095.

9. R. H. Middleton, J. H. Braslavsky, String Instability in Classes of Linear Time Invariant Formation Control with Limited Communication Range, Trans. Aut. Contr. 55, 1519-1530, 2010. 
10. D. A. Paley, A. K. Bahrani, Critical Damping in a Kinetic Interaction Network, 2010 American Control Conference, 4628-4633, 2010.

11. A. Pant, P. Seiler, K. Hedrick, Mesh Stability of Look-Ahead Systems, IEEE Trans. Aut. Contr. 47, No2, 403-408, 2002.

12. L. E. Peppard, String Stability of Relative PID Vehicle Control Systems, IEEE Trans Autom Contr, 1974, 579-581.

13. C. Robinson, Dynamical Systems, Stability, Symbolic Dynamics, and Chaos, $2^{\text {nd }}$ ed, CRC Press, 1999.

14. W. Sullivan, Boundary Conditions and a One Lane Linear model of Traffic Flow, Portland State University, Master's Thesis, 2010.

15. D. Swaroop, J. K. Hedrick, C. C. Chien, P. Ioannou, A Comparison of Spacing and Headway Control Laws for Automativally Controlled Vehicles, Vehicle System Dynamics, 23, 597-625, 1994.

16. D. Swaroop, J. K. Hedrick, String Stability of Interconnected Systems, Trans. Aut. Contr. 41, No 3, 349-357, 1996.

17. D. Swaroop, J. K. Hedrick, Constant Spacing Strategies for Platooning in Highway Systems, J. Dyn. Sys., Measurement, and Contr. 41, No 3, 462-470, 1999.

18. F. M. Tangerman, J. J. P. Veerman, B. D. Stošić, "Asymmetric Decentralized Flocks", Trans. Autom. Contr., Vol 57, Issue 11, 2844-2853, 2012.

19. L. N. Trefethen, A. E. Trefethen, S. C. Reddy, T. A. Driscoll, Hydrodynamic Stability without Eigenvalues, Science, Vol 261, 30 July 1993, 578-584.

20. L. N. Trefethen, Pseudospectra of Linear Operators, SIAM REV. Vol. 39, No. 3, pp. 383-406, September 1997.

21. J. J. P. Veerman, John S. Caughman, G. Lafferriere, A. Williams, Flocks and Formations, J. Stat. Phys. 121, Vol 5-6, 901-936, 2005.

22. J. J. P. Veerman, Stability of Large Flocks: an Example, arXiv:1002.0768, 2009.

23. J. J. P. Veerman, F.M Tangerman, Impulse Stability of Large Linear Flocks: an Example, arXiv:1002.0782, 2009.

24. J. J. P. Veerman, Symmetry and Stability of Homogeneous Flocks (a Position Paper), Proc. 1st Int'l Conf. on Pervasive and Embedded Computing and Communication Systems, Algarve, 2010.

25. J. J. P. Veerman, B. D. Stosic, F. M. Tangerman, Automated Traffic and The Finite Size Resonance, J. Stat. Phys. 137, Issue 1, 189-203, 2009.

26. S. K. Yadlapalli, S. Darbha, K. R. Rajagopal, Information Flow and Its Relation to Stability of the Motion of Vehicles in a Rigid Formation, IEEE Trans. Autom. Contr., Vol 51, No 8, 1315-1319, 2006.

27. N. W. Ashcroft, N. D. Merman, Solid State Physics, Harcourt College Publishers, 1976. 